GEOMETRY IN NONLINEAR CONTROL

AND DIFFERENTIAL INCLUSIONS

BANACH CENTER PUBLICATIONS, VOLUME 32

INSTITUTE OF MATHEMATICS

POLISH ACADEMY OF SCIENCES

WARSZAWA 1995

\title{
FORWARD INVARIANT SETS, HOMOGENEITY AND SMALL-TIME LOCAL CONTROLLABILITY
}

\author{
MIKHAIL KRASTANOV \\ Department of Operations Research, Institute of Mathematics \\ Bl. Acad. G. Bonchev 8, 1113 Sofia, Bulgaria
}

\begin{abstract}
The property of forward invariance of a subset of $\mathbf{R}^{n}$ with respect to a differential inclusion is characterized by using the notion of a perpendicular to a set. The obtained results are applied for investigating the dependence of the small-time local controllability of a homogeneous control system on parameters.
\end{abstract}

1. Introduction. The problem of small-time local controllability (STLC) at a point $x$ is an important topic in control theory because:

- it can be viewed as a particular case of the general problem of obtaining higher order optimality conditions;

- it is equivalent to the continuity property of the optimal time function at $x$, etc.

Necessary and sufficient conditions are known only for linear (cf. [14], [17], etc.), piecewise linear (cf. [19]) and so called "odd systems" (cf. [1]). Some general sufficient conditions (cf. [16], [6], [2], etc.) as well as some necessary conditions (cf. [12], [15], [8], [10], etc.) are obtained for nonlinear control systems.

In this paper we characterize the property of forward invariance of a subset of $\mathbf{R}^{n}$ with respect to a differential inclusion in terms of the notion of "perpendiculars" to a set. Basing on the obtained results we investigate how the STLC property of a well-known control system (cf. [12], [9]) depends on parameters.

2. Forward invariant sets. Let us consider a system described by the differential inclusion

$$
\dot{x} \in F(x), \quad x \in \mathbf{R}^{n},
$$

1991 Mathematics Subject Classification: 93B03, 93B05.

Key words and phrases: differential inclusions, forward invariant sets, homogeneous control systems, small-time local controllability.

The paper is in final form and no version of it will be published elsewhere. 
under the following assumptions:

A1. The multivalued mapping $F: \mathbf{R}^{n} \rightarrow \mathbf{R}^{n}$ is compact valued;

A2. For every compact set $K \subset \mathbf{R}^{n}$, there exists a positive real $L$ such that

$$
H(F(x), F(y)) \leq L\|x-y\| \quad \text { for any points } x, y \in K,
$$

where $H(A, B)$ denotes the Hausdorff distance between the subsets $A$ and $B$ of $\mathbf{R}^{n}$ defined by $H(A, B)=\max \left(H^{+}(A, B), H^{+}(B, A)\right)$, where

$$
H^{+}(A, B)=\inf \{\alpha \geq 0: A \subset B+\alpha B(0,1)\}
$$

and $B(x, r) \subset \mathbf{R}^{n}$ is the ball with center $x$ and radius $r$.

A3. The multivalued mapping $F(\cdot)$ has the Lipschitz-selection property (cf. [11], $[4])$, i.e. for each $x \in \mathbf{R}^{n}$ and for each $v \in F(x)$, there exist a compact neighborhood $U$ of $x$ and a Lipschitz continuous mapping $f: U \rightarrow \mathbf{R}^{n}$ such that $f(x)=v$ and $f(y) \in F(y)$ for every $y \in U$.

We say that an absolutely continuous function $x(\cdot)$, defined on the interval $[0, T], T>0$, is an admissible trajectory of (1) if $x(t)$ satisfies (1) for almost every $t$ on $[0, T]$. By $\mathcal{R}(x, T), T>0$, we denote the reachable set of (1) from the point $x$ at time $T$, i.e. $\mathcal{R}(x, T)$ is the set of all points $y$ of $\mathbf{R}^{n}$ which can be reached from $x$ at time $T$ by means of trajectories of (1). The system (1) is said to be small time local controllable (STLC) at the point $x$ if $x$ belongs to the interior of the set $\mathcal{R}(x, T)$ for every time $T>0$.

Definition 2.1. A set $M \subset \mathbf{R}^{n}$ is forward invariant with respect to the system (1) if every solution of (1) starting from a point of $M$ remains in $M$.

Definition 2.2. A set $M \subset \mathbf{R}^{n}$ is complete forward invariant with respect to the system (1) and to the point $x \in \mathbf{R}^{n}$ iff:

(i) $M$ is forward invariant with respect to (1);

(ii) every point $y \in M$ is reachable from the point $x$ by means of a trajectory of (1) which lies in $M$.

Let us define a multivalued mapping $H: \mathbf{R}^{n} \times \mathbf{R}^{n} \Rightarrow \mathbf{R}$ as follows:

$$
H(p, x)=\{\langle p, v\rangle: v \in F(x)\},
$$

where $\langle.,$.$\rangle denotes the standard scalar product in \mathbf{R}^{n}$ and let $H^{*}(p, x)=\max \{r$ : $r \in H(p, x)\}$.

For $M \subset \mathbf{R}^{n}$ we denote

$$
\mathcal{P}_{M}(x)=\{y \in \operatorname{cl} M:\|x-y\|=\operatorname{dist}(x, M)\},
$$

where cl $M$ denotes the closure of $M$ and $\operatorname{dist}(x, M)$ denotes the distance between the point $x$ and the set $M$, i.e.

$$
\operatorname{dist}(x, M)=\inf _{y \in M}\|x-y\|
$$

(here $\|$.$\| denotes the standard Euclidian norm in \mathbf{R}^{n}$ ). We say that $p \in \mathbf{R}^{n}$ is a perpendicular to $M$ at $x$ if $x \in \mathcal{P}_{M}(x+\alpha p)$ for some $\alpha>0$ (cf. [5]). We denote 
by $N_{M}^{\perp}(x)$ the set of all unit perpendiculars to $M$ at $x \in \partial M$ ( $\partial M$ denotes the boundary of $M$ ). This set can be empty at some points $y \in \partial M$.

A characterization of the viability property of a target in terms of "perpendiculars" is presented in [18]. In this paper we prove the following

THeOREM 2.1. The closed set $M$ is forward invariant with respect to (1) if and only if

$$
\sup _{p \in N_{M}^{\perp}(y)} H^{*}(p, y) \leq 0 \quad \text { for every } y \in \partial M .
$$

Remark 1. In fact, it is proved that if the set $M$ is forward invariant (but not closed), then the relation (2) is also satisfied.

Pr o of. First we prove that the relation (2) is necessary for forward invariance. Let $M$ be a forward invariant set with respect to the system (1) and let us suppose that (2) is not true. This means that there exist a positive number $\rho$ and a point $y \in \partial M$ such that $N_{M}^{\perp}(y) \neq \emptyset$ and

$$
\sup _{p \in N_{M}^{\perp}(y)} \max _{v \in F(y)}\langle p, v\rangle=\rho>0 .
$$

Thus we can find $p \in N_{M}^{\perp}(y)$ and $v \in F(y)$ such that

$$
\langle p, v\rangle>\rho / 2 .
$$

According to A3, there exists a compact neighborhood $U$ of $y$ and a Lipschitz continuous mapping $f: U \rightarrow \mathbf{R}^{n}$ such that $f(y)=v$ and $f(z) \in F(z)$ for every point $z \in U$. We can find a positive $T$ and a compact neighborhood $V$ of $y$ such that for every point $z_{0} \in V$ the differential equation

$$
\dot{z}=f(z)
$$

has a solution $z\left(., z_{0}\right)$, starting from the point $z_{0} \in V$, defined on $[0, T]$ and belonging to $U$.

The relation (3) implies that $z(T, y)$ does not belong to $\mathrm{cl} M$ for every $T>0$ which is sufficiently small. Since the solutions of (4) depend on the starting point $z_{0}$ in a continuous way, it follows that also $z\left(T, z_{0}\right)$ does not lie in $M$ for every point $z_{0}$ which belongs to a sufficiently small neighborhood of $y$. But this means that there exists a solution of (4) starting from a point of $M$ which leaves $M$ at time $T$. But this is impossible. So the forward invariance of the set $M$ implies the relation (2).

Next we prove that the relation (2) is sufficient for forward invariance. Let (2) be satisfied. Let $z(\cdot)$ be an arbitrary trajectory of (1) starting from the point $z \in M$ and defined on $[0, T]$. Setting $d(t)=\operatorname{dist}(z(t), M), t \in[0, T]$, we define an absolutely continuous function.

The compactness of the curve

$$
Z=\{z(t): t \in[0, T]\}
$$


implies compactness of the set

$$
B(Z, r)=\left\{y \in \mathbf{R}^{n}: \operatorname{dist}(y, Z) \leq r\right\},
$$

where $m$ is an arbitrary chosen point of $M$ and

$$
r=\max (\|z(t)-m\|: t \in[0, T]) .
$$

Conditions A1 and A2 imply the existence of positive reals $C, L$ such that for every point $z$ from $B(Z, r)$,

$$
\begin{gathered}
\max \{\|v\|: v \in F(z), z \in B(Z, r)\} \leq C, \\
H\left(F\left(z_{1}\right), F\left(z_{2}\right)\right) \leq L\left\|z_{1}-z_{2}\right\| \quad \text { for } z_{1}, z_{2} \in B(Z, r) .
\end{gathered}
$$

Suppose $z(\cdot)$ and $d(\cdot)$ are differentiable at the point $t$ (this is true for almost all $t$ ).

Suppose $z(t)$ does not belong to cl $M$. Applying theorem 2.3.9 and proposition 2.5.4 from [5] we obtain

$$
\dot{d}(t)=\left\langle\frac{z(t)-q(t)}{\|z(t)-q(t)\|}, \dot{z}(t)\right\rangle, \quad \text { where } q(t) \in \mathcal{P}_{M} z(t) .
$$

We know that $\dot{z}(t) \in F(z(t))$ and

$$
p(t)=\frac{z(t)-q(t)}{\|z(t)-q(t)\|} \in N_{M}^{\perp}(q(t)) .
$$

Since

$$
\|z(t)-q(t)\|=\operatorname{dist}(z(t), M) \leq\|z(t)-m\| \leq r
$$

we get $q(t) \in B(Z, r)$. Let us choose $v \in F(q(t))$ in such a way that

$$
\|v-\dot{z}(t)\| \leq 2 H(F(q(t), F(z(t)) .
$$

This implies (according to (2) and (6)) that

$$
\begin{aligned}
\|\dot{d}(t)\| & =\langle p(t), \dot{z}(t)\rangle \leq\langle p(t), v\rangle+\langle p(t), \dot{z}(t)-v\rangle \\
& \leq 0+2 H(F(z(t)), F(q(t))) \leq 2 L\|z(t)-q(t)\| \\
& =2 L \operatorname{dist}(z(t), M)=2 L d(t) .
\end{aligned}
$$

So when $z(t)$ does not belong to $\mathrm{cl} M$ we have

$$
\|\dot{d}(t)\| \leq 2 L d(t) .
$$

If $z(t) \in \operatorname{int} M$, then $\|\dot{d}(t)\|=0$.

Let $z(t) \in \operatorname{cl} M$. Then propositions 2.5.4 and 2.5.6 of [5] imply that the estimate $(7)$ is also true.

Since $d(t)$ satisfies the relations $d(0)=0, \dot{d}(t) \leq 2 L d(t)$ for almost every $t \in[0, T]$ we conclude that $d(t)=0$ for $t \in[0, T]$, i.e. $z(t) \in \operatorname{cl} M$ for $t \in[0, T]$. But this means that $M=\mathrm{cl} M$ is a forward invariant set with respect to (1). This completes the proof. 
THEOREM 2.2. Let $M \subset \mathbf{R}^{n}$ be a complete forward invariant set with respect to (1) and to a point $x \in M$. Then

$$
\sup _{p \in N_{M}^{\perp}(y)} H^{*}(p, y)=0 \quad \text { for every } y \in \partial M
$$

( for which $N_{M}^{\perp}(y) \neq \emptyset$ ).

Proof. Let us assume that (8) does not hold. Since $M$ is forward invariant with respect to the control system (1), theorem 2.1 implies that

$$
\sup _{p \in N_{M}^{\perp}(y)} H^{*}(p, y) \leq 0
$$

for every $y \in \partial M$ (for which $N_{\bar{M}}^{\perp}(y) \neq \emptyset$ ). Hence, our assumption means (according to (9)) that there exist a point $y \in \partial M$ and a positive number $\rho$ such that $N_{M}^{\perp}(y) \neq \emptyset$ and

$$
\sup _{p \in N_{M}^{\perp}(y)} \max _{v \in F(y)}\langle p, v\rangle=-\rho<0 .
$$

The following cases are possible:

Case I: $M=\{x\}$. The assumption implies that there exists an element $v \in$ $F(x),\|v\| \neq 0$, and this implies existence of a solution of the differential inclusion that leaves $M$. But this contradicts the forward invariance of $M$. Therefore, case I is impossible.

Case II: $M \supset\{x\}$ and $y \in \partial M, y \neq x$. Let us fix an arbitrary element $p \in N_{M}^{\perp}(y)$. According to (10),

$$
\max _{v \in F(y)}\langle p, v\rangle \leq-\rho
$$

The assumptions A1 and A2 imply existence of a neighborhood $V$ of $y$ such that for any point $z$ from $V$,

$$
\max _{v \in F(z)}\langle p, v\rangle \leq-\rho / 2 \quad \text { and } \quad \max \{\|v\|: v \in F(z), z \in V\} \leq C .
$$

Let us define the set

$$
K=\left\{z \in R^{n}:\langle p, z\rangle \geq(A-\delta)\|z\|\right\},
$$

where $A=\frac{\rho}{2 C}$, and $0<\delta<A$.

Claim 1. There exist a constant $D>0$ such that for every $0<\mu<D$ and for every $z \in K,\|z\|=1$, the point $y+\mu z$ does not belong to $M$.

Proof. The definition of a perpendicular to a set implies that there exists a point $y^{\prime}=y+\left\|y^{\prime}-y\right\| p$ such that $\left\|y^{\prime}-y\right\|=\operatorname{dist}\left(y^{\prime}, M\right)$. We set $D:=$ $2\left\|y^{\prime}-y\right\|(A-\delta)$. Let us fix a real $\mu, 0<\mu<D$. Then for every element $z \in K$, $\|z\|=1$, we have $\langle p, z\rangle \geq(A-\delta)\|z\|$ and hence

$$
\begin{aligned}
\left\|y^{\prime}-y-\mu z\right\|^{2} & =\left\langle y^{\prime}-y-\mu z, y^{\prime}-y-\mu z\right\rangle=\left\|y^{\prime}-y\right\|^{2}-2 \mu\left\langle y^{\prime}-y, z\right\rangle+\mu^{2}\|z\|^{2} \\
& =\left\|y^{\prime}-y\right\|^{2}-2 \mu\left\|y^{\prime}-y\right\|\langle p, z\rangle+\mu^{2}
\end{aligned}
$$




$$
\begin{aligned}
& \leq\left\|y^{\prime}-y\right\|^{2}-2 \mu\left\|y^{\prime}-y\right\|(A-\delta)\|z\|+\mu^{2} \\
& =\left\|y^{\prime}-y\right\|^{2}-\mu\left(2\left\|y^{\prime}-y\right\|(A-\delta)-\mu\right)<\left\|y^{\prime}-y\right\|^{2} .
\end{aligned}
$$

Since $\left\|y^{\prime}-y\right\|:=\operatorname{dist}\left(y^{\prime}, M\right)$ the above inequality implies that $y+\mu z$ does not belong to $M$. This completes the proof of claim 1 .

Claim 2. Let $q \in \mathbf{R}^{n} \backslash K,\|q\|=1, \mu>0$ and $0<\varepsilon<\mu \min \left(1, \frac{\delta}{1+\rho /(2 C)}\right)$. Let $z(\cdot)$ be an arbitrary solution of (1) starting from the point $y+\mu q$ and defined on $[0, T]$ such that $z(t) \in V$ for every $t \in[0, T]$. Then $\|y-z(t)\| \geq \varepsilon$ for every $t \in[0, T]$.

Pr o of. Let $z(\cdot)$ be a solution of (1) starting from the point $y+\mu q$ and defined on $[0, T]$ such that $z(t) \in V$ for every $t \in[0, T]$.

If $0<t<\frac{\mu-\varepsilon}{C}$ then

$$
\begin{aligned}
\|y-z(t)\| & =\left\|y-z(0)-\int_{0}^{T} \dot{z}(\tau) d \tau\right\| \\
& =\left\|-\mu q-\int_{0}^{T} \dot{z}(\tau) d \tau\right\| \geq \mu-\int_{0}^{T}\|\dot{z}(\tau) d \tau\| \geq \mu-t C>\varepsilon .
\end{aligned}
$$

Since $q \notin K$ we have that $\langle p, q\rangle<(A-\delta)\|q\|$. Moreover, the equalities $A=\frac{\rho}{2 C}$, $\|q\|=1$, the choice of $\varepsilon$ and (11) imply that if $t \geq \frac{\mu-\varepsilon}{C}$ then

$$
\begin{aligned}
\|y-z(t)\| & \geq\langle p, y-z(t)\rangle \\
& =-\mu\langle p, q\rangle-\int_{0}^{t}\langle p, \dot{z}(\tau) d \tau\rangle>\mu\left(\delta-\frac{\rho}{2 C}\right)\|q\|+\frac{t \rho}{2} \\
& \geq \mu\left(\delta-\frac{\rho}{2 C}\right)+\frac{(\mu-\varepsilon) \rho}{2 C}=\mu \delta-\frac{\varepsilon \rho}{2 C}>\varepsilon .
\end{aligned}
$$

Both considered cases complete the proof of claim 2.

Let us fix a real $\mu, 0<\mu<D$, such that $x \notin B(y, \mu) \subset V$. Since $y \in \partial M$ there exists a point $y_{\varepsilon} \in M \cap B(y, \mu)$ such that $\left\|y_{\varepsilon}-y\right\|<\min (\varepsilon, \mu)$. The definition of a complete forward invariant set with respect to (1) and to $x$ implies that there is a solution $z_{\varepsilon}(\cdot)$ of $(1)$ defined on an interval $\left[0, T_{\varepsilon}\right]$ such that

$$
z(0)=x, \quad z\left(T_{\varepsilon}\right)=y_{\varepsilon}, \quad z(t) \in M, \quad t \in\left[0, T_{\varepsilon}\right] .
$$

The continuity of $z(\cdot)$ and our choice of $y_{\varepsilon}$ imply that the compact subset

$$
N_{T}=\left\{t \in\left[0, T_{\varepsilon}\right]:\|z(t)-y\|=\mu\right\}
$$

of $\left[0, T_{\varepsilon}\right]$ is not empty. Let $\bar{t}=\max \left\{t: t \in N_{T}\right\}$. Then $z(t) \in B(y, \mu) \cap V$ for every $t \in\left[\bar{t}, T_{\varepsilon}\right]$. We set

$$
q:=\frac{z(\bar{t})-y}{\mu} .
$$


If $q \in K$, then claim 1 implies that $z(\bar{t})=y+\mu q \notin M$. But this is impossible. Hence $q \in \mathbf{R}^{n} \backslash K$. Then according to claim 2 we obtain

$$
\left\|y-y_{\varepsilon}\right\|=\left\|y-z\left(T_{\varepsilon}\right)\right\| \geq \varepsilon,
$$

which contradicts the choice of $y_{\varepsilon}$.

Case III: $\quad M \supset\{x\}$ and $y=x$. Let us fix an arbitrary element $p \in N_{M}^{\perp}(x)$ and let $x^{\prime} \notin M$ be such that $x \in \mathcal{P}_{M}\left(x^{\prime}\right)$ and $p=\frac{x^{\prime}-x}{\left\|x^{\prime}-x\right\|}$. As in case II, one can prove the existence of a compact neighborhood $V$ of $x$ such that

$$
\max _{v \in F(z)}\langle p, v\rangle \leq-\rho / 2
$$

for every point $z \in V$. We define the set

$$
L=\left\{z \in \mathbf{R}^{n}:\langle p, z\rangle \geq A\|z\|\right\},
$$

where $A:=\frac{\rho}{2 C}$. It can be directly checked that $L$ is a convex closed cone in $\mathbf{R}^{n}$.

Claim 3. There exists a positive $T$ such that every solution $z(\cdot)$ of (1) starting at $x$ and defined in $[0, T]$ satisfies the inequality

$$
\langle p, x-z(t)\rangle \geq t \rho / 2 .
$$

Proof. Without loss of generality (according to A1 and A2), we may think that

Let us fix a real $T>0$ such that

$$
\max \{v \in F(z): z \in V\} \leq C .
$$

$$
\left\{z \in \mathbf{R}^{n}:\|z-x\| \leq C T\right\} \subset V .
$$

Let $z(\cdot)$ be an arbitrary solution of (1) starting from $x$ and defined on $[0, T]$. Then for every $t \in[0, T]$,

$$
\|z(t)-x\|=\left\|\int_{0}^{t} \dot{z}(\tau) d \tau\right\| \leq t C,
$$

which means that $z(t) \in V$ for every $t \in[0, T]$. But then (12) implies that

$$
\langle p, x-z(t)\rangle=-\int_{0}^{t}\langle p, \dot{z}(\tau) d \tau\rangle \geq-\int_{0}^{t} \max _{v \in F(z(t))}\langle p, v\rangle d \tau \geq \frac{t \rho}{2} .
$$

This completes the proof of claim 3 .

Since $x \in M$ and $M$ is complete forward invariant with respect to (1) and $x$, there exists a solution $z_{x}(\cdot)$ of (1) defined on some interval $\left[0, T_{x}\right]$ such that

$$
z_{x}(0)=x, \quad z_{x}\left(T_{x}\right)=x, \quad z_{x}(t) \in M, \quad t \in\left[0, T_{x}\right] .
$$

If we suppose that $T_{x} \leq T$ then claim 3 implies that $\left\|x-z_{x}\left(T_{x}\right)\right\| \geq T_{x} \rho / 2$. But this is impossible because of the relation $z_{x}\left(T_{x}\right)=x$.

Therefore $T_{x}>T$. Then the inequalities $\left\|x-z_{x}(T)\right\| \geq T \rho / 2>0$ imply the existence of a compact neighborhood $W$ of $x$ such that $z_{x}(T) \notin W$. Continuing as in case II, we find that case III is also impossible. 
So, we have considered all possible cases obtaining a contradiction in each case. This shows that our assumption (that the relation (8) is not valid) is false. This completes the proof of theorem 2.2.

3. Homogeneity and small-time local controllability. Because of the difficulties connected with the study of reachable sets of nonlinear control systems, it seems reasonable to consider some relatively simple class of them. In our opinion, one possible choice is the class of systems which are "homogeneous" with respect to some dilation. This class of nonlinear systems represents the so called "graded approximations" of smooth control systems. These approximations lead to computationally feasible results in the fields of differential equations and control theory. Roughly speaking these approximations retain information relevant to the study of "structurally stable" properties associated with the original system.

First we give some definitions which we use further (for details cf. [3], [7] etc.).

Let $m=\left(m_{1}, \ldots, m_{n}\right)^{T}$ be a vector of positive integers and $\varepsilon$ be a positive real. A map $\delta_{\varepsilon}^{m}: \mathbf{R}^{n} \rightarrow \mathbf{R}^{n}$, for which $\delta_{\varepsilon}^{m}\left(x_{1}, \ldots, x_{n}\right)=\left(\varepsilon^{m_{1}} x_{1}, \ldots, \varepsilon^{m_{n}} x_{n}\right)$, is called a dilation. A function $h: \mathbf{R}^{n} \rightarrow \mathbf{R}$ is called homogeneous of degree $s$ with respect to $\delta_{\varepsilon}^{m}$ if $h\left(\delta_{\varepsilon}^{m} x\right)=\varepsilon^{s} h(x)$ for some positive integer $s$.

Let $k \geq \max \left\{m_{i}: 1 \leq i \leq p\right\}$. The dual dilation $\delta_{k, \varepsilon}^{* m}: \mathbf{R}^{n} \rightarrow \mathbf{R}^{n}$ is defined by $\delta_{k, \varepsilon}^{* m}\left(p_{1}, \ldots, p_{n}\right)=\left(\varepsilon^{k-m_{1}} p_{1}, \ldots, \varepsilon^{k-m_{n}} p_{n}\right)$.

Let us recall the following maps (defined in the previous section):

$$
H(p, x)=\{\langle p, v\rangle: v \in F(x)\} \quad \text { and } \quad H^{*}(p, x)=\max \{r: r \in H(p, x)\},
$$

where $x \in \mathbf{R}^{n}$ and $p \in \mathbf{R}^{n}$.

DEFinition 3.1. $H^{*}$ (as well as the underlying control system) is homogeneous with respect to $\left(\delta_{\varepsilon}^{m}, \delta_{k, \varepsilon}^{* m}\right)$ if $H^{*}\left(\delta_{k, \varepsilon}^{* m} p, \delta_{\varepsilon}^{m} x\right)=\varepsilon^{s} H^{*}(p, x)$ for some positive integer $s$.

Next we show how to apply the obtained results for studying the STLC property of homogeneous control systems at the origin. The main idea is the following: We look for a complete forward invariant set with respect to the control system and to the origin of the following type:

$$
M=\left\{x \in \mathbf{R}^{n}: G(x) \leq 0\right\},
$$

where $G: \mathbf{R}^{n} \rightarrow \mathbf{R}$ is a suitable chosen function.

The homogeneity of $H^{*}$ with respect to the dilation $\delta_{\varepsilon}^{m}(x)$ and its dual dilation $\delta_{k, \varepsilon}^{* m}(p)$ implies that we should seek a homogeneous solution of (8) (cf. [7], p. 259). This means the following: Assuming that $y \in \partial M$ and that $G$ is analytic in an open neighborhood of $y$, we look for a function $G$ which could be represented as a finite linear combination of monomials homogeneous of degree $k$ with respect to $\delta_{\varepsilon}^{m}$. Let $d G(y)$ denote the gradient of $G$ at the point $y$. Assuming that $d G(y) \neq 0$, we have $d G(z) \neq 0$ when $z$ belongs to some neighborhood $\Gamma_{y}$ of $y$. Since in this 
case $N_{M}^{\perp}(z)=d G(z)$, the relation (8) becomes

$$
H^{*}(d G(z), z)=0, \quad z \in \Gamma_{y} \cap \partial M .
$$

From this relation we could try to find the unknown function $G$. Suppose that there exists a locally Lipschitz function which coincides with the homogeneous functions found as above on the corresponding neighborhoods $\Gamma$. Applying the next proposition 3.1, we could prove the forward invariance of $M$. And hence, if the origin belonged to the boundary of $M$, then this system could not be STLC at the origin.

Proposition 3.1. Let $G: \mathbf{R}^{n} \rightarrow \mathbf{R}$ be a locally Lipschitz function, $G_{i}: \mathbf{R} \rightarrow$ $\mathbf{R}, i=1, \ldots, s$, be continuously differentiable functions, $z \in \mathbf{R}^{n}$,

$$
M=\left\{x \in \mathbf{R}^{n}: G(x) \leq G(z)\right\}, \quad 0 \notin \partial G(x) \quad \text { for every } x \in M .
$$

Let us assume that if $G$ is differentiable at a point $y$ of $\partial M$, then

$$
d G(y) \in\left\{d G_{i}(y): i=1, \ldots, s\right\} \quad \text { and } \quad H^{*}\left(d G_{i}(y), y\right) \leq 0, \quad i=1, \ldots, s .
$$

Then $M$ is forward invariant with respect to (1).

Proof. Let $y$ be an arbitrary point of the set $\partial M$ such that $N_{M}^{\perp}(y) \neq \emptyset$ and $p$ be an arbitrary element of the set $N_{M}^{\perp}(y)$. Then proposition 2.5.7 and corollary 1 of theorem 2.4.7 in [5] imply that there exist $\lambda>0$ such that $\lambda p \in \partial G(y)$. From theorem 2.5.1 of [5] we see that there exist positive reals $\alpha_{i}>0, i=1, \ldots, \beta$, $\sum_{i=1}^{\beta} \alpha_{i}=1$ and sequences $\left\{y_{i}^{\mu}\right\}_{\mu=1}^{\infty}, \lim _{\mu \rightarrow \infty} y_{i}^{\mu}=y, i=1, \ldots, \beta$, such that $\lambda p=\sum_{i=1}^{\beta} \alpha_{i} \lim _{\mu \rightarrow \infty} d G\left(y_{i}^{\mu}\right)$. Then (15) implies that

$$
\begin{aligned}
\max _{v \in F(y)} \lambda\langle p, v\rangle & =\lambda \max _{v \in F(y)} \sum_{i=1}^{\beta} \alpha_{i}\left\langle\lim _{\mu \rightarrow \infty} d G\left(y_{i}^{\mu}\right), v\right\rangle \\
& \leq \lambda \sum_{i=1}^{\beta} \alpha_{i} \max _{v \in F(y)} \lim _{\mu \rightarrow \infty}\left\langle d G\left(y_{i}^{\mu}\right), v\right\rangle \\
& \leq \lambda \sum_{i=1}^{\beta} \alpha_{i} \max _{v \in F(y)} \lim _{\mu \rightarrow \infty} \max \left\{\left\langle d G_{j}\left(y_{i}^{\mu}\right), v\right\rangle: j=1, \ldots, s\right\} \\
& =\lambda \sum_{i=1}^{\beta} \alpha_{i} \max _{v \in F(y)} \max \left\{\left\langle d G_{j}(y), v\right\rangle: j=1, \ldots, s\right\} \leq 0 .
\end{aligned}
$$

Applying theorem 2.1, we complete the proof.

Rem ark 3.1. Analogously, if $G: \mathbf{R}^{n} \rightarrow \mathbf{R}$ is a locally Lipschitz function, $z \in \mathbf{R}^{n}$

$$
M=\left\{x \in \mathbf{R}^{n}: G(x) \geq G(z)\right\}, \quad 0 \notin \partial G(x) \quad \text { for every } x \in M,
$$


and assuming that if $G$ is differentiable at a point $y$ from $\partial M$, then

(17) $d G(y) \in\left\{d G_{i}(y): i=1, \ldots, s\right\} \quad$ and $\quad H^{*}\left(-d G_{i}(y), y\right) \leq 0, \quad i=1, \ldots, s$,

it can be proved that $M$ is forward invariant with respect to (1).

As an application, we investigate the STLC property of the following threedimensional polynomial control system $(\Sigma)$ :

$$
\begin{array}{llrl}
\dot{x} & =u, & x(0) & =0, \\
\dot{y} & =x, & y(0) & =0, \\
\dot{z} & =y^{2}-A x^{4}, & z(0) & =0,
\end{array}
$$

where $u \in[v, w], v<0<w$.

To our knowledge, this control system is considered in the literature only in the case $w=-v$. First it was shown that this system is STLC at the origin for sufficiently large values of $w$ (cf. [13]). In [9] it is proved that this system is not STLC at the origin when $A v^{2} \leq \frac{2}{3}$ and it is claimed that this system is STLC at the origin in the contrary case. In what follows we show what happens when $u \in[v, w], v<0<w$.

Let us assume that $w>0$ and $A w^{2}>\frac{2}{3}$ (the case when $v<0, A v^{2}>$ $\frac{2}{3}$ can be considered in the same way). Setting $m=(1,2,5)^{T}$ and $k=5$, we define the dilation $\delta_{\varepsilon}^{m}(x, y, z)=\left(\varepsilon x, \varepsilon^{2} y, \varepsilon^{5} z\right)$ and its dual dilation $\delta_{k, \varepsilon}^{* m}(p, q, r)=$ $\left(\varepsilon^{4} p, \varepsilon^{3} q, r\right)$. For this system we have

$$
H(x, y, z, p, q, r, u)=p u+q x+r\left(y^{2}-A x^{4}\right)
$$

and hence,

$$
H^{*}(x, y, z, p, q, r)= \begin{cases}p w+q x+r\left(y^{2}-A x^{4}\right) & \text { if } p \geq 0, \\ p v+q x+r\left(y^{2}-A x^{4}\right) & \text { if } p \leq 0 .\end{cases}
$$

It can be calculated that

$$
H^{*}\left(\delta_{\varepsilon}^{m}(x, y, z), \delta_{k, \varepsilon}^{* m}(p, q, r)\right)=\varepsilon^{4} H^{*}(x, y, z, p, q, r) .
$$

This means that $H^{*}$ is homogeneous of degree $s=4$ with to respect to the dilation $\delta_{\varepsilon}^{m}(x, y, z)$ and its dual dilation $\delta_{k, \varepsilon}^{* m}(p, q, r)$. For that reason we look for a complete invariant set $M$ of the form

$$
M=\{(x, y, z): G(x, y, z) \geq 0\}
$$

where $G$ is a continuous function homogeneous of degree $k=5$ with respect to the dilation $\delta_{\varepsilon}^{m}$. For this choice of $M$ we have $N_{M}^{\perp}(x, y, z)=-d G(x, y, z)$ for every point $(x, y, z) \in \mathbf{R}^{n}$ where the function $G$ is differentiable.

Assuming that $\left(x_{0}, y_{0}, z_{0}\right) \in \partial M$ and $G$ is analytic in a neighborhood $\Gamma$ of $\left(x_{0}, y_{0}, z_{0}\right)$, we represent $G$ as a linear combination of homogeneous monomials:

$$
G(x, y, z)=z+B x y^{2}+C x^{3} y+D x^{5}, \quad(x, y, z) \in \Gamma .
$$


There are two homogeneous functions $G_{w}$ and $G_{v}$ satisfying (13). We find them as follows: It can be directly checked that

$$
d G(x, y, z)=\left(B y^{2}+3 C x^{2} y+5 D x^{4}, 2 B x y+C x^{3}, 1\right),
$$

and $-d G(x, y, z)=N_{M}^{\perp}(x, y, z)$.

First we consider the case when

$$
p=-\frac{\partial G}{\partial x}\left(x_{0}, y_{0}, z_{0}\right)>0 .
$$

Then $-\partial G / \partial x(x, y, z)>0$ in a (possibly smaller) open neighborhood of $\left(x_{0}, y_{0}, z_{0}\right)$ which we denote by $\Gamma$ too. The condition (13) implies that

$$
-\left(B_{w} y^{2}+3 C_{w} x^{2} y+5 D_{w} x^{4}\right) w-\left(2 B_{w} x y+C_{w} x^{3}\right) x-\left(y^{2}-A x^{4}\right)=0,
$$

i.e.

$$
\left(B_{w}+1\right) y^{2}+\left(3 C_{w} w+2 B\right) x^{2} y+\left(5 D_{w}+2 C+A\right) x^{4}=0 .
$$

Since $x$ and $y$ take infinitely many values in $\Gamma \cap \partial M$, the last equality implies that

$$
\begin{gathered}
B_{w}=-\frac{1}{w}<0, \quad C_{w}=-\frac{2}{3 w} B_{w}=\frac{2}{3 w^{2}}, \\
D_{w}=\frac{1}{5 w}(A-C)=\frac{1}{5 w}\left(A-\frac{2}{3 w^{2}}\right) .
\end{gathered}
$$

The relation (18) reduces to the inequality

$$
B_{w} y^{2}+3 C_{w} x^{2} y+5 D_{w} x^{4} \leq 0,
$$

which is satisfied for every $w$ for which $y / x^{2}(x \neq 0)$ does not belong to the interval $\left[R_{w}^{-}, R_{w}^{+}\right]$, where

$$
R_{w}^{ \pm}=\frac{1 \pm \sqrt{\frac{1}{3}+A w^{2}}}{w} .
$$

So, if $x \neq 0$ and $y / x^{2} \notin\left[R_{w}^{-}, R_{w}^{+}\right]$then

$$
G_{w}(x, y, z)=z+B_{w} x y^{2}+C_{w} x^{3} y+D_{w} x^{5}
$$

where $B_{w}, C_{w}$ and $D_{w}$ satisfy (19).

As above, one can show that if $x \neq 0$ and $y / x^{2} \notin\left[R_{v}^{-}, R_{v}^{+}\right]$, where

$$
R_{v}^{ \pm}=\frac{1 \pm \sqrt{\frac{1}{3}+A v^{2}}}{v} .
$$

then

$$
G_{v}(x, y, z)=z+B_{v} x y^{2}+C_{v} x^{3} y+D_{v} x^{5},
$$

where $B_{v}, C_{v}$ and $D_{v}$ satisfy the relations

(21) $B_{v}=-\frac{1}{v}<0, \quad C_{v}=-\frac{2}{3 v} B_{v}=\frac{2}{3 v^{2}}, \quad D_{v}=\frac{1}{5 v}(A-C)=\frac{1}{5 v}\left(A-\frac{2}{3 v^{2}}\right)$. 
We set

$$
s=\sqrt{\frac{1}{3}+A w^{2}}>1, \quad K=\frac{1-s}{w}<0, \quad v_{c}=\frac{w}{2-3 S}>\frac{1}{2 K},
$$

and consider the following cases:

Case I: $0>v \geq v_{c}$. We assert that in this case the control system $(\Sigma)$ is not STLC at the origin. Clearly, it is sufficient to prove this only for the case $v=v_{c}$. The proof is based on remark 3.1. We construct a locally Lipschitz continuous function $G_{c}$ by setting

$$
G_{c}(x, y, z)= \begin{cases}G_{w}(x, y, z) & \text { if } y \leq K x^{2} \\ G_{v}(x, y, z) & \text { if } y \geq K x^{2}\end{cases}
$$

If $G_{c}$ is differentiable at the point $(x, y, z)$ then

$$
d G(x, y, z) \in\left\{d G _ { w } \left((x, y, z), d G_{v}((x, y, z)\}\right.\right.
$$

and therefore $\frac{\partial G}{\partial z}(x, y, z)=1$. According to theorem 2.5.1 of [5] we find that $0 \notin \partial G(x, y, z)$ for every point $(x, y, z) \in \mathbf{R}^{3}$.

Since the roots of the equation $z^{2}-2 z / w+1 / w^{2}\left(2 / 3-A w^{2}\right)=0$ are

$$
z_{1,2}=\left(1 \pm\left(1 / 3+A w^{2}\right)^{1 / 2}\right) / w
$$

and $K=z_{1}$, we have

$$
\begin{aligned}
\left\langle-d G_{w}^{T}(x, y, z),\right. & \left.\left(u, x, y^{2}-A x^{4}\right)\right\rangle \\
& =-(w-u) / w\left[y^{2}-2 y x^{2} / w+x^{4} / w^{2}\left(2 / 3-A w^{2}\right)\right] \leq 0
\end{aligned}
$$

for every $(x, y, z)$ such that $y \leq K x^{2}$. Taking into account that $A w^{2}=s-1 / 3$, it can be calculated that

$$
K=\left[1-\left(1 / 3+A v_{c}^{2}\right)^{1 / 2}\right] / v_{c} .
$$

Since the roots of the equation $z^{2}-2 z / v_{c}+1 / v_{c}{ }^{2}\left(2 / 3-A v_{c}{ }^{2}\right)=0$ are

$$
z_{1,2}=\left(1 \pm\left(1 / 3+A v_{c}^{2}\right)^{1 / 2}\right) / v_{c},
$$

and $K=z_{2}$ (cf. (23)) we have

$$
\begin{aligned}
\left\langle-d G_{v_{c}}^{T}(x, y, z)\right. & \left.,\left(u, x, y^{2}-A x^{4}\right)\right\rangle \\
= & -\left(v_{c}-u\right) / v_{c}\left[y^{2}-2 y x^{2} / v_{c}+x^{4} / v_{c}{ }^{2}\left(2 / 3-A v_{c}{ }^{2}\right)\right] \leq 0
\end{aligned}
$$

for every $(x, y, z)$ such that $y \geq K x^{2}$.

The inequalities (22) and (24) show that the inequalities for $H^{*}$ of remark 3.1 are satisfied.

The relation $G_{w}\left(x, K x^{2}, z\right)=G_{v_{c}}\left(x, K x^{2}, z\right)$ implies the continuity of $G_{c}$. So, we found that $G_{c}(\cdot)$ is a continuous piecewise analytic function (hence, it is locally Lipschitz) such that $0 \notin \partial G(x, y, z)$ for every point $(x, y, z) \in \mathbf{R}^{3}$. According to remark 3.1 we conclude that the control system $(\Sigma)$ is not STLC at the point 0 .

Case II: $v<v_{c}$. We show that the control system $(\Sigma)$ is STLC at the origin. Let $q(\cdot)$ be an arbitrary periodic trajectory for which the control $u$ satisfies the 
following feedback law:

$$
u(x, y, z)= \begin{cases}w & \text { if } y \leq K x^{2} \\ v & \text { if } y>K x^{2}\end{cases}
$$

It can be directly checked that the function $G_{c}$ is strictly decreasing along $q(\cdot)$. Then we construct the following admissible trajectory:

(i) using the control function $u(\cdot) \equiv w$ we start from the point $(0,0,0)$ and reach the periodic trajectory $q(\cdot)$;

(ii) using the control satisfying the feedback law defined above we make $N$ turnovers along the periodic trajectory $q(\cdot)$;

(iii) using the control $u(\cdot) \equiv v$ and starting from a suitable point of the periodic trajectory $q(\cdot)$, we reach a point $P=\left(x_{p}, y_{p}, z_{p}\right)$ for which $x_{p}=y_{p}=0$.

It can be checked directly that $z_{p}<0$ for sufficiently large values of $N$. It is trivial to show that there exist $A_{ \pm}, B_{ \pm}, A>0$ and $B>0$ such that the points

$$
(0,0, A), \quad\left( \pm A, 0, A_{ \pm}\right), \quad\left(0, \pm B, B_{ \pm}\right)
$$

belong to the reachable set starting from the origin for some positive time. Then lemma 3 of [2] implies that the control system $(\Sigma)$ is STLC at the origin.

In such a way we proved the following

Proposition 3.2. Let us consider the control system $(\Sigma)$.

(i) If $A v^{2} \leq \frac{2}{3}$ and $A w^{2} \leq \frac{2}{3}$ then $(\Sigma)$ is not $S T L C$ at the origin.

(ii) Let $A w^{2}>\frac{2}{3}$. We set

$$
S_{w}=\sqrt{\frac{1}{3}+A w^{2}}>1, \quad K_{w}=\frac{1-S_{w}}{w}<0, \quad 0>v_{c}=\frac{w}{2-3 S_{w}}>\frac{1}{2 K_{w}} .
$$

Then

- if $v<v_{c}$ the system $(\Sigma)$ is STLC at the origin;

- if $v \geq v_{c}$ the system $(\Sigma)$ is not STLC at the origin.

(iii) Let $A v^{2}>\frac{2}{3}$. We set

$$
S_{v}=\sqrt{\frac{1}{3}+A v^{2}}>1, \quad K_{v}=\frac{1-S_{v}}{v}>0, \quad 0<w_{c}=\frac{v}{2-3 S_{v}}<\frac{1}{2 K_{v}} .
$$

Then

- if $w>w_{c}$ the system $(\Sigma)$ is STLC at the origin;

- if $w \leq w_{c}$ the system $(\Sigma)$ is not STLC at the origin.

\section{References}

[1] P. Brunovský, Local controllability of odd systems, in: Banach Center Publ. 1, PWN Warszawa, 1976, 39-45.

[2] R. Bianchini and G. Stefani, Sufficient conditions on local controllability, in: Proc. 25th IEEE Conf. Decision \& Control, Athens, 1986, 967-970. 
[3] - - - Graded approximations and controllability along a trajectory, SIAM J. Control Optim. 28 (1990), 903-924.

[4] - - - Self-accessibility of a set with respect to a multivalued field, J. Optim. Theory Appl. 31 (1980), 535-552.

[5] F. Clarke, Optimization and Nonsmooth Analysis, Wiley, New York, 1983.

[6] H. Hermes, Control systems with generate decomposable Lie algebras, J. Differential Equations 44 (1982), 166-187.

[7] -, Nilpotent and high-order approximations of vector field systems, SIAM Rev. 33 (1991) $238-264$.

[8] M. Kawski, A necessary condition for local controllability, Contemp. Math. 68 (1987), $143-155$.

[9] - , High-order small time local controllability, in: Nonlinear Controllability and Optimal Control, H. Sussmann (ed.), Marcel Dekker, New York, 1990, 431-467.

[10] M. Krastanov, A necessary condition for local controllability, C. R. Acad. Bulgare Sci. 41 (7) (1988), 13-15.

[11] C. Lobry, Sur l'ensemble des points atteignables par les solutions d'une équation différentielle multivoque, Publ. Math. Bordeaux 1 (5) (1973).

[12] G. Stefani, On the local controllability of a scalar input control system, in: Theory and Applications of Nonlinear Control Systems, C. Byrnes and A. Lindquist (eds.), Elsevier Science Publ., 1986, 167-179.

[13] - Polynomial approximation to control systems and local controllability, in: Proc. 25th IEEE Conf. on Decision \& Control, Ft. Landerdale, 1985, 33-38.

[14] H. Sussmann, Small-time local controllability and continuity of the optimal time function for linear systems, J. Optim. Theory Appl. (1988), 281-297.

[15] -, Lie brackets and local controllability: a sufficient condition for scalar-input systems, SIAM J. Control Optim. 21 (1983), 686-713.

[16] -, A general theorem on local controllability, ibid. 25 (1987), 158-194.

[17] V. Veliov, On the controllability of control constrained linear systems, Math. Balkanica $2(2-3)(1988), 147-155$.

[18] - On the Lipschitz continuity of the value function in optimal control, to appear.

[19] V. Veliov and M. Krastanov, Controllability of piecewise linear systems, Systems Control Lett. 7 (1986), 335-341. 